\title{
A Natural Language Generation Technique for Automated Psychotherapy
}

\author{
Graham Mann ${ }^{(凶)}(\mathbb{D})$, Beena Kishore (D), and Pyara Dhillon (D) \\ Murdoch University, 90 South Street Murdoch, Perth, WA 6150, Australia \\ \{g.mann, b.kishore, p.dhillon\} @murdoch. edu . au
}

\begin{abstract}
The need for software applications that can assist with mental disorders has never been greater. Individuals suffering from mental illnesses often avoid consultation with a psychotherapist, because they do not realize the need, or because they cannot or will not face the social and economic consequences, which can be severe. Between ideal treatment by a human therapist and self-help websites lies the possibility of a helpful interaction with a language-using computer. A model of empathic response planning for sentence generation in a forthcoming automated psychotherapist is described here. The model combines emotional state tracking, contextual information from the patient's history and continuously updated therapeutic goals to form suitable conceptual graphs that may then be realized as suitable textual sentences.
\end{abstract}

Keywords: Natural language generation $\cdot$ Conceptual graphs $\cdot$ Model-based reasoning

\section{Introduction}

Many parts of the world now face a serious mental health care treatment gap, especially in low to middle income countries, and non-urban areas in high income countries [1]. The reasons are complex, but much of the shortage is caused by a lack of available skilled psychiatric professionals, and a failure of engagement by patients for economic or social stigma reasons [2]. A review of evidence shows that there are good reasons to think computerized therapy may be one effective approach to overcoming these difficulties [3]. While we do not imagine that these would be equivalent to consultation with skilled human psychiatrists, even existing mental health care apps can play a role and would often be better than nothing. In the case of "talking" therapies - those relying primarily on psychiatric interviews - software can today carry out natural conversations with a patient, simulating the role of the therapist. This paper deals with the formation and expression of appropriate responses to be used by an automated therapist during a consultation. It is a conceptual graph (CG) based language theory realized as a computer model of language generation called Affect-Based Language Generation (ABLG).

Current trends in conversational systems tend to favour machine learning (ML) approaches, typically employing neural networks $(\mathrm{NN})$, but we believe that these are not ideal in this application, for the following reasons. First, the knowledge and executable 
skills of a machine learning system are typically opaque, lack auditability and so lack trust [4]. This is a serious drawback in medical applications. Knowledge and skills in conceptual graph (CG) based systems are as a rule much more human-readable and subject to logical reasoning that can readily be comprehended and verified. Second, NNbased or statistical ML approaches (with the possible exception of Bayesian learners) cannot easily incorporate high level, a priori knowledge into their processing [5]. This disadvantages learners in domains where such high-level knowledge is available or must be policy. But by virtue of their standardized knowledge representation, CG systems can freely mix prior knowledge incoming data relatively easily. Third, ML language systems are typically very data-hungry, and while large corpuses of language knowledge are now available, using these is computationally expensive. By contrast, model-based CG systems can, with some labour, be made to work with a relatively small amount of domain-specific language knowledge and with little or no learning.

In the rest of this paper, Sect. 2 proposes a system model that draws on tracked emotional states, patient's utterances and background information about the patient with pragmatic cues and goals from a control executive to generate a suitable response in conceptual form. Section 3 briefly describes our experimental implementation, consisting of heuristics to fetch instances of the above informative content, and calling on conceptual functions to filter these and bring them together to form CGs that can be realised as linear texts. The whole process is controlled by an executive expert system implementing psychotherapeutic rules. Finally, Sect. 4 concludes with some current challenges of this approach and its prospects for testing and further development.

\section{Sources Informing the Generation of Responses}

Sentence generation involves the planning of conceptual content first, and then linguistically encoding it into a grammatical string of words [6]. Our idea of generating sentences is based on a therapeutic process informed by representations of the patient's current emotional state, representations of their pre-clinical interview history, and representations of their on-going utterances.

\subsection{Tracking of Patient's Expressed Emotions}

It is difficult to imagine a successful psychotherapist who is not concerned with the emotional state of the patient. Even behaviourist therapies that emphasise overt actions in response to stimuli over mental state today include emotions as a recognised behavioural response, if not an important internal state determining them [e.g., 7]. The evidence is clear that the patient's emotional state which is important for treatment needs to be closely monitored [8]. This state must be dealt with properly to maintain patients in a comfortable place, while at the same time empathizing, noting the significance of the emotion and helping the patient to find meaning from it. Much emotional information can be obtained by monitoring a speaker's tone of voice, facial expression or other body language. Today's mobile devices, with their microphones and cameras could hope to read these forms of expression, but since at this stage our work is about testing a theory of natural language generation, not a practical app, we use only text. 
According to the survey conducted by Calvo and D'Mello [9] on models of affect, early approaches to detect emotional words in text include lexical analysis of the text to recognize words that are immanent of the affective states [10] or specific semantic analyses of the text based on an affect model [11]. The current work adapts Smith \& Ellsworth's six-dimensional model [12] to make a system that can better grasp the subtleties of patient affect. Their chosen modal values on the principle component states for 15 distinguished emotional states are shown in Table 1.

Table 1. Mean locations of labelled emotional points in the range $[-1.5,+1.5]$ as compiled in Smith \& Ellsworth's study.

\begin{tabular}{l|r|r|r|r|r|r}
\hline Emotion & P & R & C & \multicolumn{1}{l}{ A } & E & O \\
\hline Happiness & -1.46 & 0.09 & -0.46 & 0.15 & -0.33 & -0.21 \\
\hline Sadness & 0.87 & -0.36 & \multicolumn{1}{l}{0} & -0.21 & -0.14 & 1.15 \\
\hline Anger & 0.85 & -0.94 & -0.29 & 0.12 & 0.53 & -0.96 \\
\hline Boredom & 0.34 & -0.19 & -0.35 & -1.27 & -1.19 & 0.12 \\
\hline Challenge & -0.37 & 0.44 & -0.01 & 0.52 & 1.19 & -0.2 \\
\hline Hope & -0.5 & 0.15 & 0.46 & 0.31 & -0.18 & 0.35 \\
\hline Fear & 0.44 & -0.17 & 0.73 & 0.03 & 0.63 & 0.59 \\
\hline Interest & -1.05 & -0.13 & -0.07 & 0.7 & -0.07 & 0.41 \\
\hline Contempt & 0.89 & -0.5 & -0.12 & 0.08 & -0.07 & -0.63 \\
\hline Disgust & 0.38 & -0.5 & -0.39 & -0.96 & 0.06 & -0.19 \\
\hline Frustration & 0.88 & -0.37 & -0.08 & 0.6 & 0.48 & 0.22 \\
\hline Surprise & -1.35 & -0.97 & 0.73 & 0.4 & -0.66 & 0.15 \\
\hline Pride & -1.25 & 0.81 & -0.32 & 0.02 & -0.31 & -0.46 \\
\hline Shame & 0.73 & 1.31 & 0.21 & -0.11 & 0.07 & -0.07 \\
\hline Guilt & 0.6 & 1.31 & -0.15 & -0.36 & 0 & -0.29 \\
\hline
\end{tabular}

A patient's textual utterance is compared to accumulated word-bags that offer clues to the expressed emotions, plus a filter to exclude references to the emotions of others. These classify the expressed emotion into one of the Smith \& Ellsworth's 15 ideal values, the vectors of which locate the expression as a single point in a six-dimensional affective space. This allows mappings of complex emotional states into a consistent hypervolume so that, for example, the "distances" between two states can be computed. It also allows emotive subspaces to be defined. One way that emotional tracking can be used is for the appropriate application of sympathy. We define a "safe region" in the affective space. The therapist may continue the therapy as long as the patient's tracked emotional state stays within the safe region. A single point was chosen as the "most distressed" emotional state (we used $\left\{\begin{array}{llll}1.10 & 1.3 & 1.15 & 1.0-1.15 \\ 2.0\end{array}\right\}$ ). The simplest model of a safe region is outside a hypersphere of fixed radius centred on this point. The process is then reduced to finding the Euclidian distance between the current emotional state and the above-defined distressed centre. 


$$
\Delta \Omega=\sqrt{(\mathcal{P} \mathfrak{i}-\mathcal{P} \mathfrak{j})^{2}+(E \mathfrak{i}-E \mathfrak{j})^{2}+(C \mathfrak{i}-C \mathfrak{j})^{2}+(A \mathfrak{i}-A \mathfrak{j})^{2}+(R \mathfrak{i}-R \mathfrak{j})^{2}+(O \mathfrak{i}-O \mathfrak{j})^{2}}
$$

If the calculated distance is greater than an arbitrarily defined tolerance threshold (radius), the patient's current emotional state is considered safe. The calculated $\Delta \Omega$ of

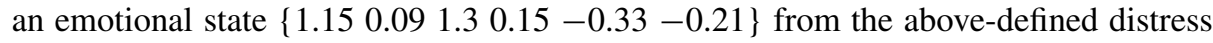
point would be 1.70 . For an arbitrary tolerance radius of 2.5 units from the distress point, the patient's tracked emotive state would not be in the safe region. A more sophisticated approach would be to map examples of real patient distress into a convex volume of the emotional space and then measure the current tracked emotional state to the nearest point on that volume.

\subsection{Conceptual Analysis of Patient's Utterances}

Study of a reference corpus of 118 talking therapy interviews [13], reveals that these patient utterances can be long and rambling, often incoherent and quite difficult for a person, much less a machine, to comprehend. While we have a conceptual parser, SAVVY, capable of converting real, non-grammatical paragraphs into meaning-preserving CGs [14], it was not developed for use in this domain. For the present work we do not intend to improve it to the point of creating meaningful conceptual representations for most of the utterances observed in our corpus. Conceptual parsers depend on an ontology in the form of a hierarchy of concepts, a set of relations and a set of actors. Manually creating representations of all the terms used in those interviews for SAVVY would be a difficult and time-consuming task. (This most serious of drawbacks for conceptual knowledgebased systems is now being addressed in automated ontology-building machines [e.g. 15]). Our focus in this study is the generation of language. Yet this kind of psychotherapy is essentially conversational, so we must allow the conceptual representations of patient utterances to be an input even to test response formation. Therefore, SAVVY will be adapted to accept selected patient utterances of interest. In some cases, to keep the project manageable, we hand-write plausible input CGs to avoid diverting too much time and energy away from our generation pipeline.

\subsection{Using Context to Inform the Planning Process}

In regular clinical practice, the first step for a new patient is an admitting (or triage) interview, that can capture important biographical details, a presenting complaint, background histories, and perhaps an initial diagnosis. Because we wish our model of language generation to account for existing, contextual information, we will not actively model this initial interview, but rather only subsequent interviews that have access to this previously gathered background. A set of background topics that should be sought during an admitting interview is described by Morrison [16]. Our current model draws 12 topics from this source and adds three extra topics specific to our clinical model. 


\subsection{Executive Control}

An executive system based on a theory about how therapy should be done is needed for overall control. At each conversational turn, the executive should recommend the best "pragmatic move" and therapeutic goal for the response. This allows for the selection and instantiation of appropriate high-level conceptual templates that form the therapist's utterances to support, guide, query, inform or sympathize with the patient as appropriate during the treatment process. Our executive is based on the brief therapy of Hoyt [17] and the solution-based therapy of Shoham et al. [18]. As recommended by Hoyt, the focus is on negotiating treatment practices, not diagnostic classification. However, in this experiment a working diagnosis might become available as a result of the therapy or be input as background knowledge.

For a natural interviewing style, the executive must allow its goal-seeking behaviour to be interrupted by certain imperatives imposed by conversational conventions and good clinical practice. If the patient asks a question, this deserves some kind of answer. If the patient wishes to express some attitude or feeling about some point, that should usually be entertained immediately. If the patient's estimated emotional state falls into distress, it is important that the treatment model is suspended until the patient can be comforted and settled. Similarly, if rapport with the patient is lost (the quality of the patient's responses deteriorates), special steps must be taken to recover this before anything else can be done. We call these forced responses, to distinguish them from less obligatory pragmatic moves, which in our model are driven by key goals in the therapy.

In most cases, a conceptual structure representing a suitable therapist's response can be formed by unifying pragmatically selected schemata with content-bearing information from the other sources. This process is to be handled by heuristic rules that must be sufficiently general to keep the number needed as low as possible. In a few cases, a single standardized expressive form can be accessed without the need for unification.

\subsection{Response Generation Architecture}

The proposed architecture of the ABLG system relies on three principle processes (Fig. 1): Preparing input for Therapeutic Expert, the Therapeutic Expert System, and the Surface Realization System. Based on the input sources, heuristic tests set the values of key variables controlling the behaviour of the Therapeutic Expert, such as patient type, clarity of the patient's chief complaint, the patient's readiness to change, their current emotional state, and their rapport with the therapist. At each conversational turn, the expert system recommends the best pragmatic move to the Surface Realisation System. This in turn chooses a feature structure template based on the pragmatic move recommended by the expert system. The template slot filler will fill in the template with relevant content, drawn from CG representation of the patient's recent utterances, or looked up from the background database. Lastly, YAG (Yet Another Generator) [19] realization library will convert the feature structure into a grammatically correct sentence for output. In some instances the Therapeutic Expert System will recommend a canned response, which can be directly output without using the Surface Realisation System. 


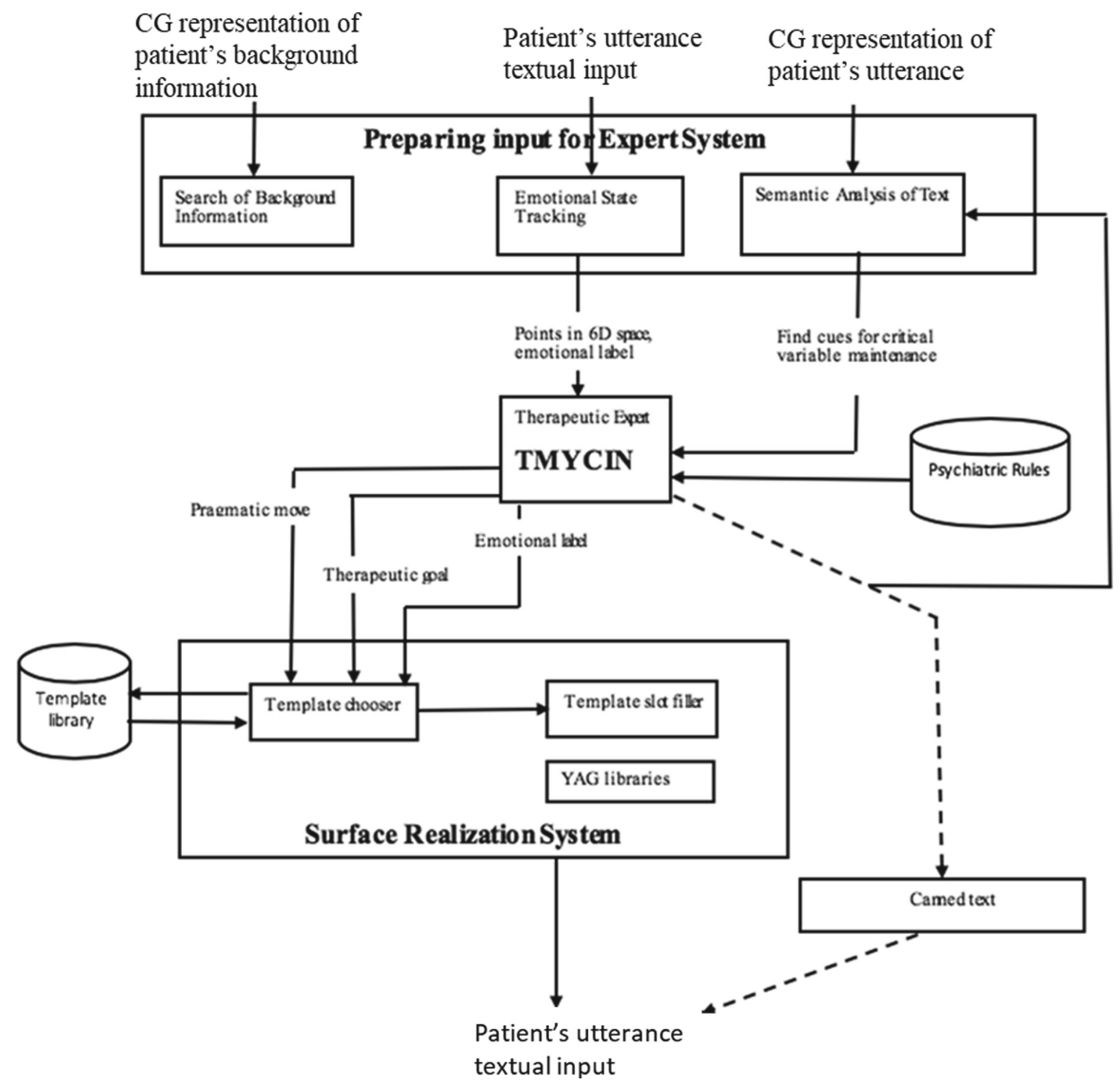

Fig. 1. Architecture of Affect Based Language Generation (ABLG) system

\section{Implementation Details}

To track emotions, we are experimenting with computationally "cheap" heuristics (meaning that, relative to machine learning approaches, logical rules on CGs do not consume very many CPU cycles). that can distinguish the patient's current emotional states directly from the text, though this has the disadvantage that it does not model cognitive aspects of emotion. To bring patient's conversational utterances into the picture, a text-to-CG parser is required. But even if it was feasible to construct complete representations for every utterance performed by a patient, this would not be desirable, because from analysis of the corpus, surprisingly few such representations would actually have useful implications for treatment, at least within our simplified model. Our conceptual parser, SAVVY, can do this because it assembles composite CGs out of prepared conceptual components that are already pre-selected for the domain of use to which they will be put.

A simple database currently provides background knowledge for our experiments. Each entry in the knowledgebase is a history list of zero or more CGs, indexed by 
both a patient identifier and one of the 15 background topics (Sect. 2.3) such as suicide_attempts, willingness_to_change and chief_complaint. Entries may be added, deleted or modified during processing, so the database can be used as a working memory to update and maintain therapeutic reasoning over sessions. Initially these entries are provided manually to represent information from the pre-existing admitting interview.

Psychiatric expertise is represented by a clinical Expert System Therapist, based on TMYCIN [20]. Consultation of the system is performed at each conversational turn, informed by the current state of variables from the inputs. Backward-chaining inference maintains internal state variables and recommends the best "pragmatic move" and "therapeutic goal". These parameters allow for the selection and instantiation of appropriate high-level templates that, when elaborated, are linearized into output texts. Further implementation details can be found in [21].

\section{Conclusion}

This generation component is still in development, so no systematic evaluation has yet been conducted. Some components have been coded and unit tested. Getting the heuristics of the system to interact smoothly with each other is a challenge; that is to be expected in this modelling approach. We are concerned about the number of templates that may be required, particularly at the surface expression level. If they become too difficult or too many to create, the method might become infeasible. The heuristic tests are not difficult to write, but are, of course, imperfect. Also, we have not fully tested the emotion tracking on many real patient texts so far.

Our planned evaluation has two parts. First, a systematic "glass-box" analysis will discover the strengths and limitations of the generation component, particularly with respect the generality of the techniques. Second, the "suitability", "naturalness" and "empathy" of the response generation for human use will be tested, using a series of ersatz patient interviews (to avoid the ethical complications of testing on real patients). Human judges (students in training to be psychotherapists) will be provided with background information and example patient utterances as well as the actual responses generated by the system. The judges will then rate these transcripts on those variables using their own knowledge of therapy. Finally, we reiterate that if hand-built conceptual representations can be practically built up using existing methods, the effort will be worthwhile if the systems are then more transparent and auditable than NN or statistical ML system and thus, more trustworthy.

\section{References}

1. Jack, H.E., et al.: Mutual capacity building to reduce the behavioral health treatment gap globally. Adm. Policy Mental Health (2019). https://doi.org/10.1007/s10488-019-00999-y

2. Meltzer, H.E. et. al.: The reluctance to seek treatment for neurotic disorders. Intl. Rev. Psychiat. 15(1-2), 123-128 (2003)

3. Fairburn, C.G., Patel, V.H.: The impact of digital technology on psychological treatments and their dissemination. Behav. Res. Ther. 88, 19-25 (2017)

4. Marcus, G.: Deep learning: a critical appraisal. arXiv preprint arXiv:1801.00631 (2018) 
5. Pearl, J.: Theoretical impediments to machine learning with seven sparks from the causal revolution. arXiv preprint arXiv:1801.04016 (2018)

6. Reiter, E., Dale, R.: Building applied natural language generation systems. Nat. Lang. Eng. 3(1), 57-87 (1997)

7. Ellis, A.: Rational-Emotive Therapy. Big Sur Recordings, pp. 32-44, CA, USA (1973)

8. Greenberg, L.S., Paivio, S.C.: Working with Emotions in Psychotherapy, vol. 13. Guilford Press, New York (2003)

9. Calvo, R.A., D'Mello, S.: Affect detection: an interdisciplinary review of models, methods, and their applications. IEEE Trans. Affect. Comput. 1, 18-37 (2010)

10. Hancock, J.T., Landrigan, C., Silver, C.: Expressing emotion in text-based communication. In: Proceedings of the SIGCHI Conference on Human Factors in Computing Systems, pp. 929932. Association for Computing Machinery (2007)

11. Gill, A.J., French, R.M., Gergle, D., Oberlander, J.: Identifying emotional characteristics from short blog texts. In: 30th Annual Conference of the Cognitive Science Society, pp. 2237-2242. Cognitive Science Society, Washington, DC (2008)

12. Smith, C.A., Ellsworth, P.C.: Attitudes and social cognition. J. Pers. Soc. Psychol. 48(4), 813-838 (1985)

13. McNally, A. et. al.: Counseling and Psychotherapy Transcripts, Volume II. Alexander Street Press, Alexandria (2014)

14. Mann, G.A.: Control of a navigating rational agent by natural language. Unpublished Ph.D. thesis, University of New South Wales, Sydney, Australia (1996). https://manualzz.com/ doc/42762943/control-of-a-navigating-rational-agent-by-natural-language. Accessed 15 Jan 2020

15. Leuzzi, F., Ferilli, S., Rotella, F.: ConNeKTion: a tool for handling conceptual graphs automatically extracted from text. In: Catarci, T., Ferro, N., Poggi, A. (eds.) IRCDL 2013. CCIS, vol. 385, pp. 93-104. Springer, Heidelberg (2014). https://doi.org/10.1007/978-3-642-543470_11

16. Morrison, J.: The First Interview: A Guide for Clinicians. Guilford Press, New York (1993)

17. Hoyt, M.F.: The temporal structure of therapy. In: O'Donohue, W.E. et al. (ed.) Clinical Strategies for Becoming a Master Psychotherapist, pp. 113-127. Elsevier (2006)

18. Shoham, V., Rohrbaugh, M., Patterson, J.: Problem-and solution-focused couple therapies: the MRI and Milwaukee models. In: Jacobson, N.S., Gurman, A.S. (eds.) Clinical Handbook of Couple Therapy, pp. 142-163. Guilford Press, New York (1995)

19. Channarukul, S., McRoy, S.W., Ali, S.S.: Enriching partially-specified representations for text realization using an attribute grammar. In: Proceedings of the 1st International Conference on NLG, vol. 14, pp. 163-170. Association for Computational Linguistics. Mitzpe Ramon, Israel (2000)

20. Novak, G.: TMYCIN expert system tool. Technical report AI87-52, Computer Science Department, University of Texas at Austin (1987). http://www.cs.utexas.edu/ftp/AI-Lab/tech-rep orts/UT-AI-TR-87-52.pdf. Accessed 5 Feb 2018

21. Mann, G., Kishore, B., Dhillon, P.: Conceptual reasoning for generating automated psychotherapeutic responses. In: Alam, M., Braun, T., Yun, B. (eds.) ICCS 2020. LNCS (LNAI), vol. 12277, pp. 186-194. Springer, Cham (2020). https://doi.org/10.1007/978-3-030-57855$8 \_14$ 
Open Access This chapter is licensed under the terms of the Creative Commons Attribution 4.0 International License (http://creativecommons.org/licenses/by/4.0/), which permits use, sharing, adaptation, distribution and reproduction in any medium or format, as long as you give appropriate credit to the original author(s) and the source, provide a link to the Creative Commons license and indicate if changes were made.

The images or other third party material in this chapter are included in the chapter's Creative Commons license, unless indicated otherwise in a credit line to the material. If material is not included in the chapter's Creative Commons license and your intended use is not permitted by statutory regulation or exceeds the permitted use, you will need to obtain permission directly from the copyright holder.

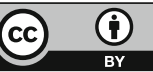

